\title{
THE EXOSAT RESULTS DATABASE
}

\author{
A.N. PARMAR AND N.E. WHITE \\ Space Science Department of ESA, ESTEC, The Netherlands
}

\begin{abstract}
The EXOSAT database provides on-line access to the results and data products (spectra, images and lightcurves) from the EXOSAT mission as well as EINSTEIN SSS and MPC spectra, EINSTEIN HRI images and IUE spectra of X-ray sources. In addition, a number of well-known optical, infrared, and X-ray catalogs, including the HST guide star catalog are available. The complete database is located at the EXOSAT observatory at ESTEC in the Netherlands and is accessible remotely via SPAn by typing 'set host $28703^{\prime}$ ' and logging in to account 'XRAY'. Alternatively, a sub-set of the database is available at GSFC and may be accessed by typing 'set host 6467 '. Access is also possible via Arpanet/Internet, GTE TELENET and by direct mail.

The database management system has been specifically developed to efficiently access the database and to allow the user to perform statistical studies on large samples of astronomical objects as well as to retrieve information on single sources. The system has been written to be mission independent and includes timing, image processing and spectral analysis package as well as software to allow the easy transfer of analysis results and products to the user's own institute.

Further information about the system including user's guides is available from A.N. Parmar and N.E. White at ESTEC and from their colleagues J. Behnke/Code 634 and K. Arnaud/Code 666 at GSFC.
\end{abstract}

Y. Kondo (ed.), Observatories in Earth Orbit and Beyond, 462.

(C)1990 Kluwer Academic Publishers. Printed in The Netherlands. 